\title{
L'âge de la Corne (1882-1887) : analyse historique, épistémologique et archéologique d'un production suisse de falsifications lacustres
}

L'âge de la Corne (1882-1887): an historical, epistemologic and archeological analysis of a Swiss prehistoric pile dwellings' forgery

\section{Cloé Lehmann}

\section{(c) OpenEdition Journals}

\section{Édition électronique}

URL : http://journals.openedition.org/artefact/826

DOI : $10.4000 /$ artefact.826

ISSN : 2606-9245

\section{Éditeur :}

Association Artefact. Techniques histoire et sciences humaines, Presses universitaires du Midi

\section{Édition imprimée}

Pagination : 77-91

ISBN : 978-2-7535-7305-5

ISSN : 2273-0753

Référence électronique

Cloé Lehmann, « L'âge de la Corne (1882-1887) : analyse historique, épistémologique et archéologique d'un production suisse de falsifications lacustres », Artefact [En ligne], 6 | 2017, mis en ligne le 31 mai 2018, consulté le 06 mars 2020. URL : http://journals.openedition.org/artefact/826 ; DOI : https:// doi.org/10.4000/artefact.826 


\section{L'âge de la Corne (1882-1887) : analyse historique, épistémologique et archéologique d'une production suisse de falsifications lacustres:}

Cloé LEHMANN ${ }^{*}$

\section{Résumé}

L'âge de la Corne correspond à une production de faux archéologiques en os et en bois de cerf, polis et décorés de motifs géométriques caractéristiques. Ceux-ci ont été réalisés dans les années 1880 sur les rives du lac de Neuchâtel (Suisse) lors d'une intense phase d'exploitation des sites palafittiques. Ce phénomène, représentatif du développement et du succès des falsifications lacustres à la fin du XIX ${ }^{e}$ siècle, est ici étudié selon trois angles d'analyse : l'histoire, l'épistémologie et l'archéologie.

Mots-clés : âge de la Corne, falsification, fraude, matières osseuses, sites lacustres, Suisse.

\section{Abstract. L'âge de la Corne (1882-1887) : an historical, epistemologic and archeological analysis of a Swiss prehis- toric pile dwellings' forgery}

L'âge de la Corne concerns a production of forgery, made from animal bones and red deer antlers, polished and decorated with characteristic geometric motifs. The objects were made during the 1880's on the shores of Neuchâtel Lake in Switzerland, while prehistoric pile dwellings around were discovered and massively exploited. This case, representative of the develop-

\footnotetext{
*. Cloé Lehmann est titulaire d'un master en archéologie de l'université de Neuchâtel (Suisse). Pour son travail de mémoire, elle a étudié une production de faussaire, l'âge de la Corne, liée à la découverte et à l'exploitation intensive des sites palafittiques à la fin du XIX ${ }^{e}$ siècle. Ses recherches lui ont permis de porter un regard interdisciplinaire en conciliant l'histoire de la recherche et les méthodes de l'archéologie. Contact : [cloe.lehmann@gmail.com].
} 
ment and the success of forgeries at the end of the 19th century, is studied by focusing analysis on history, epistemology and archaeology.

Keyword : âge de la Corne, bone material, forgery, fraud, prehistoric pile dwellings, Switzerland.

L'identification du vrai et du faux a été un moteur du développement de l'archéologie préhistorique, dès ses débuts au milieu du XIx $x^{e}$ siècle. Les célèbres affaires de fraude qui jalonnent l'histoire de la recherche - la mâchoire de MoulinQuignon, l'homme de Piltdown, le cas de Glozel - ont indirectement favorisé le développement de cette science naissante, en incitant les chercheurs d'alors à se doter d'outils méthodologiques pour démasquer le faux². En écho à l'affaire de Glozel, le préhistorien français André Vayson de Pradenne consacre, en 1932, un ouvrage à la thématique des faux en archéologie préhistorique. En quête de cas d'étude, il contacte le conservateur du musée historique de Neuchâtel, Paul Vouga, à propos d'un prétendu âge de la Corne dont la découverte, durant les années 1880, a secoué les recherches lacustres en Suisse, plus particulièrement dans la région des Trois-Lacs (Neuchâtel, Bienne et Morat ${ }^{3}$ ).

Entre 1859 et 1890, la révélation de plusieurs affaires de faux sur les rives du lac de Neuchâtel, dont celle de l'âge de la Corne, ébranle la crédibilité de la préhistoire locale. Les falsifications lacustres menacent en effet de remettre en question l'ensemble des collections palafittiques régionales. Le faux agit comme un virus : il instaure et propage le doute, rend suspect tout objet s'écartant de par sa morphologie ou ses décors des classifications établies. Dans les années 1880, la question de l'âge de la Corne entraîne la conduite de plusieurs analyses menées de manière isolée et portant sur des lots distincts d'objets'.

La présente étude ${ }^{5}$ n'a pas pour but, comme ce fut le cas au moment même des faits, de prouver la falsification, mais de caractériser cette production d'un point de vue historique, épistémologique et archéologique (approches typologique et technologique). Dans un premier temps, le phénomène de l'âge de la Corne est replacé dans son contexte et étudié avec les outils de l'historien pour retracer les événements, identifier les enjeux de l'affaire, les acteurs et leur argumentaire, ainsi que les méthodes mobilisées pour démasquer la fraude. Dans un deuxième temps, l'étude épistémologique consiste en une analyse du regard porté sur l'âge de la Corne par les chercheurs contemporains de l'affaire, afin de mettre en évidence la définition archéologique de cette industrie, telle qu'elle était perçue au siècle passé. Dans un troisième et dernier temps, l'analyse archéologique porte sur un corpus de vestiges de l'âge de la Corne déposés dans divers musées et institutions suisses. Elle a pour but de caractériser cette production : formes ou catégories d'objets, décors et motifs, techniques et traces de travail. 


\section{Cadre historique}

Pour comprendre l'extraordinaire diffusion des vestiges lacustres à travers le monde, il faut remonter trente ans avant les faits del'âge de la Corne. En 1854, suite à un hiver particulièrement sec, l'abaissement du niveau des lacs en Suisse révèle sur les rivages les restes d'habitats palafittiques. À partir des vestiges découverts à Obermeilen, au bord du lac de Zurich, l'interprétation généralisée des sites que propose Ferdinand Keller, fondateur de la Société des Antiquaires de Zurich, fait rapidement l'unanimité ${ }^{\text {. }}$ Il s'agit d'une civilisation ayant vécu en des temps pré-historiques dans des villages érigés sur des plateformes surélevées au-dessus de l'eau. Cette hypothèse engendre une vision idyllique et romantique des Lacustres, celle d'un âge d'or perdu, d'un peuple pacifique et égali- taire ${ }^{7}$. Dès lors, la société de la seconde moitié du XIx ${ }^{e}$ siècle manifeste un intérêt grandissant pour ces ancêtres lacustres dont on se met activement à rechercher les vestiges et dont le quotidien est imaginé au travers de romans, peintures, pièces de théâtre. Un marché des antiquités se développe, d'importantes collections sont constituées, ainsi que des institutions spécialement dédiées à leur accueil, les premiers musées de préhistoire. Après la présentation d'objets lacustres à l'exposition universelle de Paris, en 1867, l'intérêt dépasse les frontières. Les offres d'achat alléchantes faites par divers représentants de musées européens et de riches collectionneurs étrangers démontrent leur intérêt pour ces trouvailles. La demande s'accroît et les prix suivent la tendance.

\section{La découverte d'artefacts d'un nouvel âge}

À partir de 1882, d'intrigants objets réalisés en bois de cerf et en os, polis et décorés de motifs géométriques, circulent sur le marché des antiquités. Vendus principalement par leur découvreur, un antiquaire dénommé Gottlieb Kaiser, ils suscitent l'attention des collectionneurs et amateurs d'archéologie lacustre. Selon ce dernier, cette industrie serait caractéristique d'un nouvel âge dit « de la Corne », stratigraphiquement situé sous les niveaux des âges de la Pierre et du Bronze dans les gisements qu'il a fouillés à Forel (rive sud du lac de Neuchâtel, canton de Fribourg) et à Cortaillod (rive nord du lac de Neuchâtel, canton de Neuchâtel $^{8}$ ). Les objets se démarquent des autres artefacts découverts jusqu'alors dans les habitats palafittiques par leur esthétisme, leurs formes et décors inhabituels et leur aspect extrêmement poli (fig. 17, cahier couleur). De fait, ils rencontrent un large succès sur le marché des antiquités et sont vendus en Allemagne, en Autriche et en France. En définitive, toute collection lacustre acquise entre la fin du XIX et le début $\mathrm{du} \mathrm{xx}^{\mathrm{e}}$ siècle est susceptible d'accueillir quelques pièces de l'âge de la Corne.

Toutefois, plusieurs chercheurs émettent des doutes quant à l'authenticité de cette nouvelle culture. L'idée 
même d'un âge de la Corne est porteuse d'un enjeu scientifique, car celui-ci remet en question la chronologie de la préhistoire établie à la fin du XIx $x^{\mathrm{e}}$ siècle et, indirectement, les connaissances acquises jusqu'alors9. Bien que jamais mentionnée explicitement par les acteurs de l'époque, la problématique est de taille et concourt au doute puis à l'investigation. S'engage alors une virulente polémique que l'on peut retracer grâce à de nombreux articles de presse. Les opposants mettent en avant l'abondance des trouvailles, exclusivement liées aux excavations réalisées par Kaiser. Bien que le temps passe, les gisements de Forel et de Cortaillod semblent intarissables et révèlent en effet des objets aux formes toujours plus diversifiées. Les méthodes mobilisées par deux figures locales de l'archéologie, membres de la Société d'histoire et d'archéologie de Neuchâtel (SHAN), William Wavre, conservateur des collections préhistoriques du musée de Neuchâtel, et le $D^{r}$ Victor Gross, un amateur éclairé, ne permettent pas de démontrer la falsification de l'industrie, mais de révéler certaines incohérences dans le discours archéologique de Kaiser ou dans le déroulement des fouilles. Ainsi, on relève que les fouilleurs semblent guidés sur les emplacements où les découvertes ont lieu ${ }^{10}$. Cependant, la démonstration de la découverte in situ d'objets dans les couches archéologiques perturbe fortement ceux qui s'étaient montrés jusqu'alors sceptiques ${ }^{11}$.

La SHAN organise sur le site de Cortaillod des fouilles de contrôle, en avril 1885. Or, aucun des cent vingt-cinq objets mis au jour ne ressemble à ceux de l'âge de la Corne ${ }^{12}$ ! L'étau se resserre progressivement autour de Kaiser et, en janvier 1886, une perquisition est menée à son domicile. Le faussaire semble avoir été préalablement prévenu puisque le jour même, on n'y trouve que du « rebut et les étiquettes avec prix des antiquités de la corne, encore fixées à la muraille ${ }^{13}$ ». En mai 1886, une expertise scientifique menée par l'ingénieur Guillaume Ritter, à Neuchâtel, sonne définitivement le glas de l'âge de la Corne. Sans exclure que la matière première puisse provenir de sites lacustres et donc être véritablement archéologique, l'analyse démontre en revanche que des outils métalliques ont été utilisés pour polir les objets et graver les décors qui les ornent ${ }^{14}$.

Si Kaiser semble avoir pris la mesure de ces résultats en cessant ses activités de fouille et de vente d'antiquités, les ouvriers qui travaillent pour son compte ne semblent pas s'en inquiéter. Cette imprudence les mène à leur perte, puisque trois d'entre eux sont arrêtés à Berne, en avril 1887, alors qu'ils tentent de vendre des falsifications au directeur et au curateur de l'Antiquarium (actuel Musée historique de Berne), Edmond de Fellenberg et Édouard de Jenner. La même année, un quatrième comparse usurpe la signature de William Wavre afin d'inciter Louis de Coulon, directeur du musée historique de Neuchâtel, à acquérir plusieurs objets lacustres. Ce dernier événement motive la tenue d'un procès, le 25 juillet 1887, devant le Tribunal criminel et correctionnel de la Broye qui statue sur le sort de douze coupables prévenus de " fraude, tentative de fraude, faux en écriture privée et usage de ce faux ». Les faussaires sont condamnés à des peines d'emprisonnement allant de trois jours à quatre mois ${ }^{15}$. Kaiser, bien qu'explicitement désigné 
par les autres prévenus comme le cerveau et l'organisateur de la fraude, ne se présente pas au procès et n'est pas poursuivi.

\section{La construction de la fraude : de la production de faux au concept d'un âge nouveau}

La version fournie par l'historiographie considère les ouvriers de fouille comme les créateurs des falsifications, mais leur refuse la paternité du concept de l'âge de la Corne. L'ingéniosité de la fraude et la présence d'une tablette ornée de signes d'écriture ressemblant à ceux des premiers alphabets connus incitent les auteurs de la fin du XIX ${ }^{\mathrm{e}}$ siècle et $\mathrm{du}$ début $\mathrm{du} x \mathrm{x}^{\mathrm{e}}$ à imaginer $q \mathrm{u}^{\prime}$ un personnage instruit et lettré en serait à l'origine. Kaiser est considéré comme l'organisateur, chargé de l'aspect pratique de la supercherie : mise en scène de la découverte, publicité, ventes des faux. Sans l'affirmer clairement, les auteurs de l'époque le jugent indigne d'être à l'origine de l'idée d'un âge de la Corne. Tapissier en faillite reconverti en chercheur et marchand d'antiquités lacustres, il n'appartient pas au cercle fermé des sociétés savantes dont les membres lettrés sont généralement issus de classes sociales prospères : avocats, médecins, instituteurs. Un autre personnage, resté anonyme, est considéré comme le père de l'âge de la Corne. Il s'agit du curé de Font, village situé dans le canton de Fribourg sur la rive sud du lac de Neuchâtel. Or, rien dans nos recherches n'est venu corroborer les accusations portées à son encontre ${ }^{16}$.

Les fouilleurs, de modeste condition, étaient informés du succès de certains artefacts sur le marché des antiquités puisqu'ils revendaient une partie de leurs découvertes. En conséquence, ils ont pu avoir l'idée d'en reproduire, vraisemblablement dans un but lucratif. Trois d'entre eux, les frères Roulin, auraient investi l'argent issu de la vente de faux dans la construction d'une maison, d'après le témoignage de Baptiste Mercier au cours du jugement ${ }^{17}$. Toutefois, si les noms et situations des accusés sont connus grâce au procès, la nature de leurs liens reste difficile à reconstituer, chacun cherchant à se dédouaner des faits qui lui sont reprochés en accusant les autres. Évoluant dans le même milieu, ils se connaissent et ont certainement échangé astuces et techniques de falsification. Parmi les prévenus, seul Édouard Besse dit avoir collaboré avec quelques-uns pour la fabrication de faux. Lui-même se chargeait de la cuisson des céramiques $^{18}$. Ont-ils pour autant travaillé ensemble dans des ateliers de faussaires, comme le suggèrent certains auteurs ${ }^{19}$ ? Chacun semble plutôt œuvrer à son propre compte et profit, de manière désorganisée.

Quant à Kaiser, il ne semble pas qu'il ait lui-même fabriqué de faux. D'après le témoignage d'Émile Roulin, il en aurait repéré chez certains de ses ouvriers et les aurait encouragés dans leur démarche ${ }^{20}$. Son rôle ne se cantonne pas à celui d'in- 
termédiaire de vente, puisqu'il a l'idée de faire passer certaines falsifications en os et en bois de cerf pour des artefacts issus d'une période chronologique jusqu'alors inconnue, l'âge de la Corne, terme de sa propre invention. Kaiser se serait donc inspiré d'une pratique préexistante, mais son génie tient au fait d'avoir imaginé un concept pour définir et rassembler des productions éparses afin d'en faire une prétendue nouvelle industrie préhistorique.

Dès lors, Kaiser devient le promoteur de ce nouvel âge, auquel il tente de donner une crédibilité scientifique. Ainsi, le caractère inédit des trouvailles s'expliquerait selon lui par des fouilles menées à très grande profondeur. Il utilise l'argument de la découverte in situ comme preuve irréfutable de l'authenticité des artefacts. À cette fin, il introduit durant la nuit des faux dans les niveaux archéologiques des gisements ${ }^{21}$, qu'il fait déterrer le lendemain par ses ouvriers lors de fouilles publiques auxquelles sont conviés les notables de la région. Ces derniers auraient pu par eux-mêmes extraire du sol les objets tant décriés et leur témoignage est brandi par Kaiser comme preuve de sa propre honnêteté22. En habile orateur, il joue également sur l'ambiguïté du statut de savant en matière d'archéologie lacustre, science alors en plein essor, en qualifiant d'érudits les amateurs lettrés possesseurs d'objets de l'âge de la Corne tels que le peintre Albert de Meuron ${ }^{23}$. Enfin, Kaiser avance l'argument d'autorité scientifique, en prétendant que plusieurs musées détiennent des objets de l'âge de la Corne, dont d'illustres institutions. Il affirme dans la presse locale, utilisée comme vitrine publicitaire, que le musée national de Saint-Germain-enLaye aurait reconnu l'authenticité des artefacts selon l'avis de son conservateur Alexandre Bertrand... fait démenti presque aussitôt par ce dernier ${ }^{24}$ !

\section{Du concept à une définition archéologique}

Dans le but d'établir une synthèse de l'état de la recherche, nous avons analysé le regard que les chercheurs proches des événements ont porté sur l'âge de la Corne. Ceci afin de mettre en évidence la définition archéologique de cette industrie, telle qu'elle était perçue entre la fin du $x I x^{e}$ siècle et le début du $x x^{e}$. Cette synthèse s'avère essentielle car elle permet, en délimitant un ensemble d'objets regroupés dans la littérature scientifique sous le terme d'âge de la Corne, la mise en évidence de critères distinctifs reliés à ces productions : acteurs concernés (vendeurs et acquéreurs), sites archéologiques où ont été enfouis ces curieux artefacts, matières premières privilégiées, formes et décors particuliers, etc. Ce premier ensemble peut ensuite être comparé dans un second temps à un corpus sélectionné déposé dans les musées. L'élaboration de cet état de la recherche a également engendré quelques réflexions épistémologiques sur le mode de fonctionnement des chercheurs, notamment sur l'utilisation d'une typologie strictement fonctionnelle. 
Parmi les descriptions d'objets caractéristiques de l'âge de la Corne proposées à la fin du $\mathrm{XIX}^{\mathrm{e}}$ siècle, seule l'étude de William Wavre fournit les éléments nécessaires à l'établissement d'une définition archéologique. Témoin et acteurclé de l'affaire, Wavre publie trois ans après le procès des faussaires un rapport scientifique qui présente les affaires de faux de Concise, Yverdon et de l'âge de la Corne $^{25}$. Selon ce dernier, la période de production des objets de l'âge de la Corne se situe entre 1882 et 1885 ; elle est caractérisée par des objets en os et en bois de cerf décorés de points ou cupules. Il s'agit principalement de pièces réalisées sur andouillers de bois de cerf perforés à l'extrémité en pointe. Sur certaines, une lame de hache en roche verte est emmanchée à l'extrémité la plus large. Les décors gravés sont variés : points répartis en séries, lignes longitudinales et transversales, spirales, croix, demicercles, dents de loup, hachures en série, soleils, dièses. De manière générale, les objets se caractérisent par leur aspect poli. Wavre distingue une première catégorie d'objets très foncés et luisants, uniformément polis, parfois même enduits d'huile, d'une seconde catégorie d'artefacts polis à la poussière de charbon et dont les creux des décors sont emplis de sable censé imiter du tuf lacustre.

À partir de la définition fournie par Wavre, nous avons élaboré une première typologie en relevant et en réorganisant les différentes formes et décors mis en avant, tout en reproduisant son schéma de fonctionnement (fig. 1). Wavre décrit les pièces selon leur matière première et leur catégorie d'utilisation (parure, outil, arme, indéterminé). Le critère déterminant de son classement repose sur la fonctionnalité de l'objet. Cette typologie a ensuite été confrontée à un corpus de sources décrivant ou reproduisant (dessins, planches photographiques) cent huit objets de l'âge de la Corne, afin d'en élaborer une seconde. Se restreindre aux informations fournies par les acteurs contemporains de l'affaire s'est avéré insuffisant, le nombre de faux mentionnés étant trop limité. C'est pourquoi nous incluons également des sources postérieures constituées d'échanges entre plusieurs savants (André Vayson de Pradenne, Paul Vouga et Eugène Pittard, conservateur du musée ethnographique de Genève) dans le cadre de l'affaire de Glozel et de la préparation de l'ouvrage d'A. Vayson de Pradenne entre 1925 et 1935. Les types de cette deuxième typologie (fig. 2 et 3) ne sont pas définis d'après la fonction présupposée des objets mais d'après leur morphologie. Nous avons donc volontairement occulté les termes d'extrémité active (distale) et non active (proximale) pour caractériser les extrémités. Cependant, l'utilisation de termes archéologiques s'est avérée nécessaire pour définir certains types (épingle, lame, couteau et poignard). Nous considérons que définir un objet d'après sa fonction présupposée est, dans le cas de falsifications, inopérant. Si celles-ci imitent des objets fonctionnels, elles n'ont pourtant jamais été usitées : un poignard de l'âge de la Corne, bien qu'il imite la forme d'une arme, n'a jamais été conçu pour se battre. Il imite un objet fonctionnel mais lui-même ne l'est pas : difficulté de préhension du manche, lame non tranchante, sont autant d'indices révélant une incohérence d'utilisation ou une aberration fonctionnelle. 


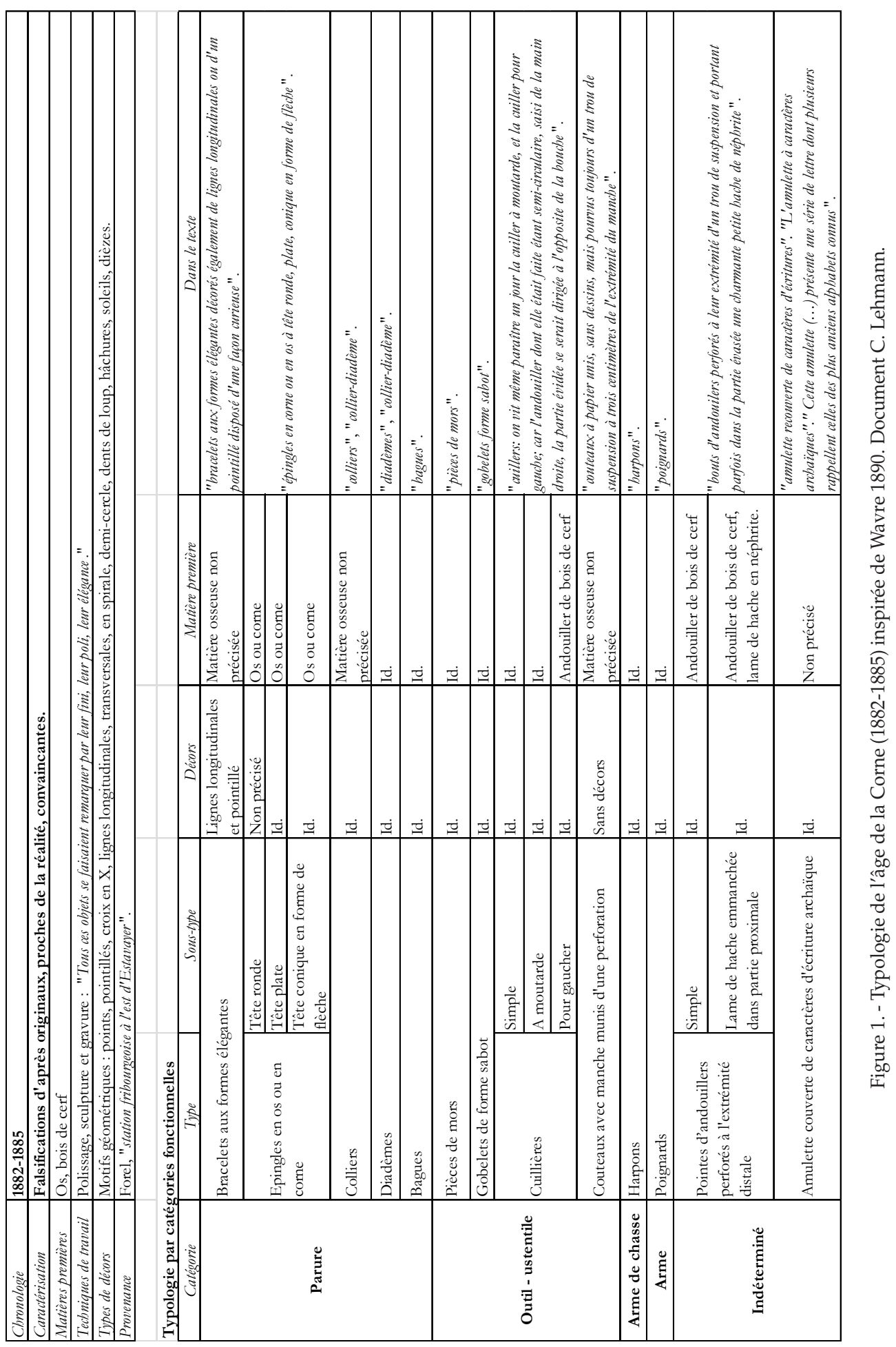


Il ne s'agit pas de remettre en question le travail de William Wavre, mais plutôt d'éclairer un aspect de la pratique archéologique de la fin du xIX ${ }^{\mathrm{e}}$ siècle, cristallisée autour de $1^{\prime}$ « obsession typologique »: classer selon un schéma évolutionniste pour construire et affiner les chronologies. Si les observations de terrain entrent dans les réflexions des chercheurs, ceuxci ne manifestent que peu d'intérêt pour la fouille, considérée comme un labeur ingrat réservé aux ouvriers. Rarement présent sur le chantier, le préhistorien s'y déplace épisodiquement pour contrôler l'avancée du travail ou faire état de découvertes importantes ${ }^{26}$. Il y a donc une séparation nette entre le travail du fouilleur, qui consiste à trouver des vestiges, et celui de l'archéologue, qui les interprète. Pour y parvenir, ce dernier doit se fier aux observations et renseignements fournis par ses manœuvres, sur le lieu de trouvaille de l'objet ou sa position stratigraphique. Dans ce contexte, la falsification des données de terrain ou l'introduction de faux sur le site est aisée. Indirectement, l'absence de cadre méthodologique et théorique pour la fouille favorise le développement de fraudes, comme cela a été démontré dans le cas des recherches de Boucher de Perthes dans les terrasses alluviales de la Somme ${ }^{27}$.

\section{L'âge de la Corne en Suisse : quels corpus?}

Dans un troisième et dernier temps, il s'agit de caractériser un corpus d'objets reconnus actuellement comme des faux de l'âge de la Corne. L'approche typologique, essentiellement descriptive, concerne l'identification et l'étude des formes ou catégories d'objets ainsi que des décors et motifs. L'approche technologique, ici en phase exploratoire, s'est attachée à l'étude des matériaux utilisés ainsi que des techniques et des traces de travail ${ }^{28}$. Premièrement, il a fallu sélectionner les critères d'étude pour construire un protocole descriptif formulé sous la forme d'une base de données : parcours de l'objet (désignation, provenance supposée, valeur, type d'acquisition), matière première et support anatomique, description de l'objet (état de conservation, forme, décors, perforations, mesures), traces et tech- niques de travail. Cette étude permet également de tester la typologie synthétisant l'état de la recherche, c'est-à-dire de confronter le regard des archéologues du XIX siècle sur l'âge de la Corne à une réalité actuelle, les objets identifiés dans les dépôts des musées.

Le choix du corpus d'étude s'est porté sur trois institutions, le Laténium, le service archéologique de l'État de Fribourg (SAEF) et le Musée national suisse ${ }^{29}$. Celles-ci sont, par l'histoire de leurs collections, directement concernées par l'affaire de l'âge de la Corne puisqu'elles sont dépositaires d'objets récoltés sur les rives de la région des Trois-Lacs dès la seconde moitié du $\mathrm{xIX}^{\mathrm{e}}$ siècle. Au total, deux cent six objets ont été réunis et étudiés à l'aide du protocole descriptif évoqué ci-dessus. 


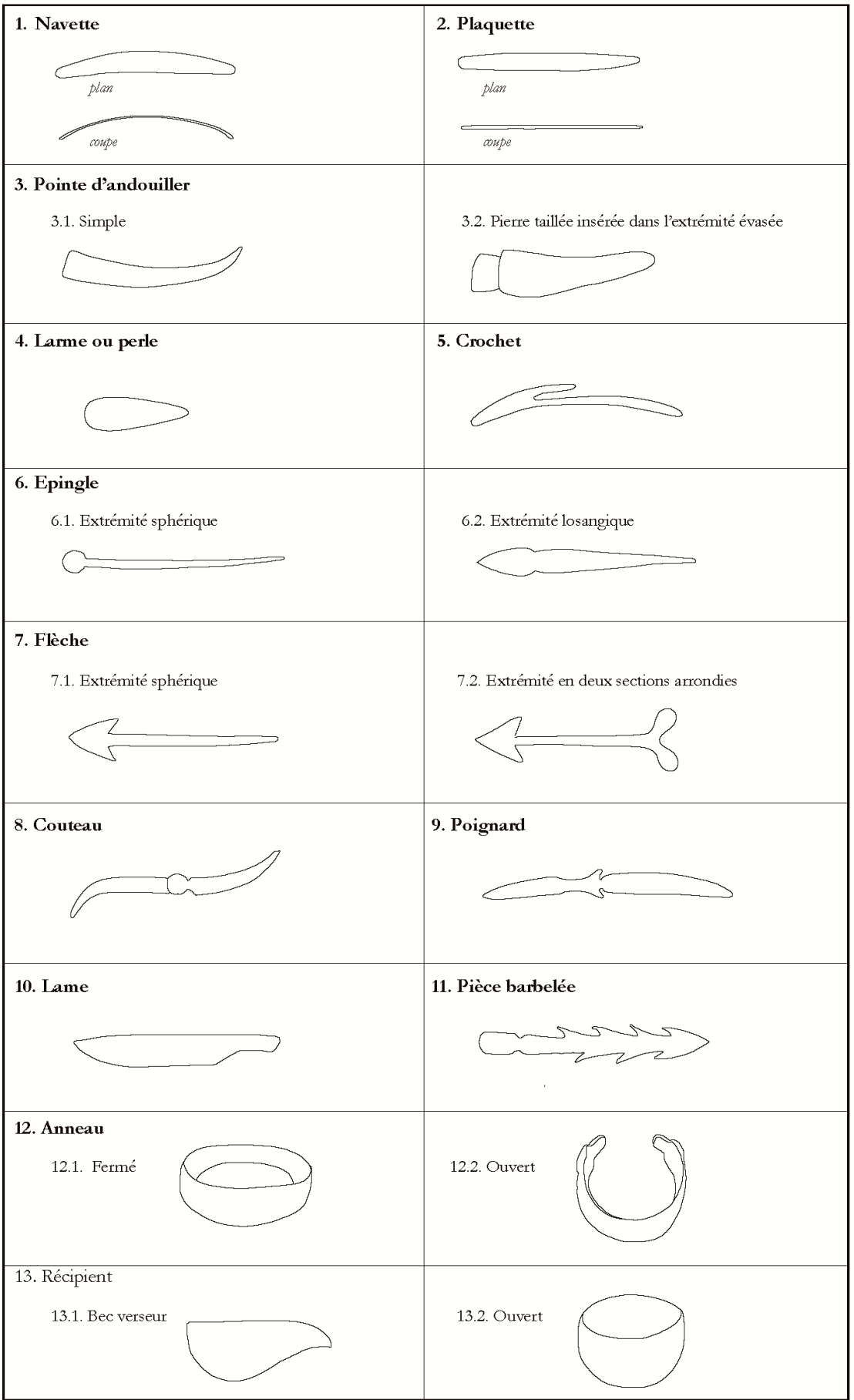

Figure 2. - Typologie morphologique des objets de l'âge de la Corne définis entre 1880 et 1935. Document C. Lehmannn. 
Parmi les principaux résultats et interprétations, il faut mentionner la difficulté de catégoriser les objets. L'idée était de reprendre les dénominations archéologiques préexistantes dans les inventaires. Cependant, au cours de l'étude, nous avons réalisé qu'il existe presque autant de dénominations que d'objets et qu'une même pièce est parfois définie selon plusieurs termes. Par exemple, au Musée national suisse, les informations fournies par l'étiquette accompagnant l'objet relevant de l'inventaire actuel diffèrent de celles fournies par l'ancien registre. Cette approche met en évidence le problème d'attribution des objets auquel on est confronté lorsqu'on tente de les inclure dans des catégories fonctionnelles. De manière simplifiée, nous avons décidé de regrouper les différents types sous six termes génériques: pointes, tranchants, éléments récepteurs et contenants, parures ou objets en suspension, masses (surface plane), indéterminés. Une comparaison des deux corpus montre qu'il existe une plus grande variabilité de formes de l'âge de la Corne, par rapport à ce qu'indique la première typologie, synthèse de l'état de la recherche entre la fin du $\mathrm{XIX}^{\mathrm{e}}$ et le début du $x x^{\mathrm{e}}$ siècle (fig. 3). En effet, sept formes identifiées lors de l'étude du corpus (aiguille, aiguille à bélière, masse, cuillère, bouton, gaine et peigne) ne présentent aucun parallèle avec la typologie élaborée.

Quant aux matériaux, le bois de cerf a servi à la réalisation de cent quatrevingt-dix objets sur un total de deux cent six et représente $92 \%$ de la matière première utilisée. On dénombre dix objets exécutés en os, sept sur os long et trois sur côte, ainsi que trois pendeloques réa- lisées sur canine de suidé. Enfin, il faut mentionner l'utilisation d'ivoire pour confectionner deux têtes d'épingle. Dans le corpus, douze objets comportent également des éléments en bronze, des tiges d'épingles et une chaîne de six anneaux insérée dans un andouiller évidé. Le bronze est parfois fortement corrodé, il pourrait s'agir d'authentiques tiges d'épingles dont la tête n'a peut-être pas été conservée, récupérées dans les couches de l'âge du Bronze de gisements palafittiques. Certaines parties anatomiques du bois de cerf sont employées à des fins précises. Par exemple, la meule a été utilisée essentiellement pour la fabrication de récipients. Il y a donc une mise à profit de la morphologie du support anatomique : en sectionnant la meule de la ramure et en évidant l'intérieur spongieux, on obtient un objet ressemblant à un récipient. De même, un court cylindre prélevé dans un merrain a servi à la réalisation de bracelets, tandis qu'un long cylindre pris dans un andouiller a servi de support pour la fabrication d'aiguilles à bélières. Tous les pendentifs ou pendeloques sont réalisés sur pointe d'andouiller. Ces exemples démontrent que, dans certains cas, le choix du support anatomique caractérise l'objet. Pour d'autres formes - harpons, pointes de flèche, épingles, aiguilles, lames, couteaux et poignards -, il est parfois difficile de déterminer si l'on a employé le merrain ou l'andouiller du bois de cerf. Si les pièces incurvées les plus allongées évoquent clairement la forme d'un andouiller, il est difficile de trancher pour celles plus courtes. Il pourrait s'agir d'un cylindre extrait sur merrain ou d'un andouiller particulièrement court évidé. 


\section{Perspectives d'une approche technologique}

Paradoxalement, un des apports les plus importants de cette étude concerne les éléments qui n'ont pas pu être observés. Il ne s'agit pas d'une limite de la méthode archéologique, bien au contraire. En effet, celle-ci met ainsi en évidence les particularités du matériel étudié, concernant notamment son analyse technologique. S'il a été possible d'identifier ou de déduire certains gestes techniques par l'observation des traces de travail, nous n'avons pas pu mettre en perspective ces gestes pour proposer une reconstitution de la chaîne opératoire. Cette impossibilité s'explique par l'absence de déchets de débitage et la présence d'objets invariablement terminés et de belle facture. Généralement, on retrouve sur les sites les déchets de mise en forme des objets, ainsi que des pièces à divers stades de réalisation (ébauches, ratés, objets abandonnés ou cassés). En outre, dans les industries archéologiques, il existe une plus grande proportion d'objets fragmentés qu'entiers, puisque ceux-ci, contrairement à ceux de l'âge de la Corne, ont été utilisés et usés à diverses tâches. Il faut toutefois garder à l'esprit qu'il ne s'agit pas d'un matériel issu de fouilles actuelles mais de collections constituées au XIX ${ }^{e}$ siècle, qui sont le fruit d'une sélection de « beaux objets » et de modifications et tris constants.

Il n'a pas été possible de déterminer de manière systématique la chronologie du polissage, des décors et perforations. Dans certains cas, les stries de polissage recoupent les rebords des gravures, mais l'inverse est aussi vrai. Parmi les techniques identifiées, celle du polissage est quasi systématique et pratiquée de manière intensive. Le polissage confère aux surfaces un aspect lisse, voire brillant. Ses traces sont discrètes, parfois invisibles à l'œil nu : il s'agit de stries fines à grossières, rectilignes et parallèles, dont l'épaisseur est probablement liée à l'utilisation d'un outil distinct ou d'un geste plus ou moins appuyé. Un travail grossier de polissage effectué sur une baguette-pendeloque (fig. 18a, cahier couleur) laisse deviner l'empreinte de l'outil utilisé, probablement une lime métallique. Enfin, cinquante-cinq objets ne présentent aucune strie, leur surface est polie si finement qu'aucune trace n'est visible à l'œil nu comme à la loupe binoculaire et l'on peut donc s'interroger sur l'outil utilisé : fibres végétales, lanière en cuir, pierre à grain fin?

Plusieurs objets ont été assemblés à l'aide de colle et teintés de manière artificielle. Contrairement aux éléments adhésifs facilement détectables, l'emploi d'un colorant assombrissant est difficilement prouvable par l'observation, à quelques exceptions. Par exemple, la zone d'emmanchement d'une pointe en bois de cerf (fig. 18c, cahier couleur) comporte quelques points de colle et est très claire par rapport à la pointe. Le bois de cerf aurait été foncé artificiellement, une fois la pointe insérée et maintenue avec de la colle dans un manche qui n'a pas été retrouvé. De même, une épingle en bois de cerf (fig. 18b, cahier couleur) présente, au niveau de l'insertion de la tige dans la tête, les traces jaune beige d'un adhésif, utilisé afin d'assembler et de maintenir les deux éléments.

Une des issues de l'étude a été de déterminer si l'âge de la Corne correspond à une « marque de fabrique », ce qui 
renvoie à l'homogénéité ou l'hétérogénéité de l'ensemble considéré. Les caractères communs de celui-ci concernent les formes et décors qui présentent une forte similarité. On retrouve, tant au Laténium qu'au SAEF ou au Musée national suisse, des objets analogues, tels que des pendeloques réalisées sur pointe d'andouiller de bois de cerf. La plupart des pièces se caractérisent par un polissage couvrant et intensif. Ce travail de finition constitue l'originalité des productions de l'âge de la Corne, l'indice d'un style particulier. Les caractères distincts concernent la disposition et l'organisation des décors qui ne présentent pas ou peu de récurrence. Si l'on retrouve les mêmes types de décors sur toutes les pièces du corpus,

\section{Conclusion}

Par cette recherche, nous avons voulu démontrer la pertinence de mêler les approches historique, épistémologique et archéologique pour l'étude d'une production de faussaires. En effet, chaque discipline fournit les informations permettant de passer à un degré supérieur d'analyse. La mise en place d'un cadre historique a permis de situer les événements, d'identifier les acteurs impliqués et $\mathrm{d}$ 'analyser les méthodes mobilisées pour dénoncer et prouver la fraude. Le déploiement de l'argumentaire des faussaires et des experts met en évidence les enjeux de l'affaire, ainsi que les motivations et intérêts de ses différentes parties prenantes. Partant de ces éléments, il est ensuite possible de réfléchir sur le processus de construction de la fraude et d'élaborer une synthèse de l'état de la recherche en ils sont toujours disposés autrement pour créer un assemblage qui semble unique à chaque objet. Plusieurs éléments attestent que l'industrie de l'âge de la Corne est le fruit d'une idée commune, partagée par plusieurs personnes, qui auraient repris un concept ou une ligne directrice et l'auraient appliqué avec quelques différences relevant de leur touche personnelle. Cette interprétation est appuyée par la présence dans le corpus de certains objets jumeaux, comme deux pendeloque-manche en forme de poisson (fig. 18d-e, cahier couleur). Ces dernières pourraient avoir été réalisées par un même faussaire ou un même groupe, tant leur ressemblance est frappante.

analysant les regards des différents chercheurs portés sur les productions de l'âge de la Corne. Ces deux premières étapes sont essentielles à mener avant d'aborder l'étude archéologique. Sans celles-ci, nous aurions en effet été incapables de reconnaître les critères distinctifs de l'âge de la Corne et de faire un tri parmi les ensembles présentés. Récurrence de certaines formes, comme des pendeloques réalisées sur pointes d'andouiller ou merrain de bois de cerf, polissage couvrant et intensif ayant laissé une surface lisse et brillante, perforations multiples et décors géométriques sont autant d'indices déterminants. Ces recherches préalables ont également amené des réflexions constructives à l'élaboration d'un protocole descriptif efficace et opérant pour une production de faussaires. 
Parmi les conséquences de l'affaire de l'âge de la Corne, il faut mentionner son action positive sur la pratique des archéologues d'alors. Ayant mobilisé les méthodes à leur disposition, ils se trouvent désemparés par l'affaire de l'âge de la Corne, puisque l'archéologie seule ne suffit pas à établir la fraude. Démunis, ils trouvent, à travers les méthodologies des sciences naturelles, les éléments permettant de prouver la fraude. Dès lors, ils intègrent les résultats de ces analyses dans leur argumentation par l'observation des objets à la loupe et l'analyse de substances modernes déposées sur les objets. En ce sens, on peut affirmer que les affaires de faux, dont celle de l'âge de la Corne, ont contribué au développement d'outils d'analyses et d'expertises des objets archéologiques et ont indirectement participé à la construction de l'archéologie en tant que discipline scientifique, en la poussant à se doter de cadres méthodologiques ${ }^{30}$. Plus intéressant encore, le succès des falsifications nous renseigne sur l'état de la recherche, la construction de la discipline, ses faiblesses et ses interrogations.

\section{Notes}

1. Nous remercions Marc-Antoine Kaeser et François-Xavier Chauvière pour leur relecture attentive.

2. Marc-Antoine KAEsER (dir.), L'âge du Faux : l'authenticité en archéologie, Hauterive, Laténium, 2011.

3. André Vayson de PRAdenne, « L'Âge de la Corne en Suisse (1882-1887) », in André VAysON DE PRADENNE, Les fraudes en archéologie préhistorique, Paris, Noury, 1932, p. 210-230; lettre de Vayson de Pradenne à Vouga, le 30 août 1928, archives du Laténium, DOC-ARCHIVE-CH A685663 et A68-5664.

4. Victor GRoss, « Ueber die eigenthümlichen Knochensnitzereien aus den Schweizer Pfahlbauten ", Mittheilungen der Anthropologischen Gesellschaft in Wien, vol. 6, 1886, p. 53-55; Louis FAVRE, "L'âge de la corne polie devant la Société des sciences naturelles de Neuchâtel », Musée neuchâtelois, 1886, p. 158-161.

5. Les recherches présentées ici sont issues d'un mémoire soutenu en septembre 2014 : Cloé LEHHMANN, « L'âge de la Corne » (1882-1887) : étude épistémologique et analyse archéologique de falsifications lacustres, mémoire de master en archéologie, université de Neuchâtel, 2014.

6. Ferdinand Keller, « Die Keltischen Pfahlbauten in den Schweizerseen », Mittheilungen des Antiquarischen Gesellschaft in Zurich, vol. 9/3, 1854, p. 65-100.

7. Marc-Antoine KAESER, Les Lacustres : archéologie et mythe national, Lausanne, Presses polytechniques et universitaires romandes, 2004.

8. Lettres de G. Kaiser publiées dans les quotidiens Gazette de Lausanne du 27 décembre 1884, $\mathrm{n}^{\circ} 07$, p. 2, et La Suisse libérale du 5 mai 1885, $\mathrm{n}^{\circ} 105, \mathrm{p} .3$. Il s'agit des seules données de terrain dont nous disposons.

9. Marc-Antoine KaEser, «L'âge de la Corne », in M.-A. KaEser (dir.), L'âge du Faux : l'authenticité en archéologie, op. cit., p. 109-111.

10. William WAVRE, «Falsifications d'antiquités lacustres », Musée neuchâtelois, 1890, p. 67-68.

11. Extrait d'une lettre de W. Wavre à M. De Tchetchermann, le 30 septembre 1884, reproduit in Nicolas PEIssARD, « L'Âge de la Corne en Pays fribourgeois ", Annales fribourgeoises, t 29, $\mathrm{n}^{\circ} 2$, 1941, p. 39.

12. Procès-verbaux de la SHAN, vol. 16841901, séance du 14 janvier 1885, p. 85-86, archives de la bibliothèque publique et universitaire de Neuchâtel. Le rapport de Vouga est publié la même année dans la revue de la Société, le Musée neuchâtelois, p. 137-140. 
13. Lettre de W. Wavre à V. Gross, le 29 janvier 1886, archives familiales Gross, Berne.

14. Procès-verbaux du Bulletin de la Société des sciences naturelles de Neuchâtel (BSSNN), vol. 15, 27 mai 1886 et 10 juin 1886, p. 227-229; Louis FAVRE, "L'âge de la corne polie devant la Société des sciences naturelles de Neuchâtel ", Musée neuchâtelois, 1886, p. 158-161.

15. Procès-verbal du procès des faussaires, le 25 juillet 1887 à Estavayer, tribunal de la Broye, affaires en matière pénale et fiscale, protocole 1885-1887, cote TBr 162, p. 567-576, archives de l'État de Fribourg.

16 W. WAVRE, « Falsifications d'antiquités lacustres », op. cit., p. 71 ; André VAYSON DE PRADENNE, op. cit., p. 228-230.

17. Procès-verbal du procès des faussaires, le 25 juillet 1887 à Estavayer, tribunal de la Broye, affaires en matière pénale et fiscale, protocole 1885-1887, cote Tbr 162, p. 570, archives de l'État de Fribourg.

18. Ibid.

19. N. Peissard, "L'Âge de la Corne en Pays fribourgeois », op. cit., p. 40; W WAVRE, "Falsifications d'antiquités lacustres ", op. cit., p. 92.

20. Procès-verbal du procès des faussaires, le 25 juillet 1887 à Estavayer, tribunal de la Broye, affaires en matière pénale et fiscale, protocole 1885-1887, cote TBr 162, p. 568, archives de l'État de Fribourg.

21. N. Peissard, «L'Âge de la Corne en Pays fribourgeois ", op. cit., p. 37; procès-verbal du procès des faussaires, le 25 juillet 1887 à Estavayer, tribunal de la Broye, affaires en matière pénale et fiscale, protocole 1885-1887, cote Tbr 162, p. 570, 572, archives de l'État de Fribourg.

22. Lettre de G. Kaiser à V. Gross, le 21 novembre 1884, archives familiales Gross, Berne.

23. Journal de Genève, n 140, 31 mai 1884, p. 1.

24. Affirmations de Kaiser parues dans La Suisse libérale, $\mathrm{n}^{\circ} 284,1^{\text {er }}$ décembre 1884 , p. 3 ; Journal de Genève, n 298, 2 décembre 1884, p. 1. Réponses de Bertrand parues dans La Suisse libérale, $\mathrm{n}^{\circ} 291$, 9 décembre 1884, p. 3 ; Journal de Genève, n 306, 11 décembre 1884, p. 1.

25 W. Wavre, "Falsifications d'antiquités lacustres », op. cit., p. 37-43, p. 67-71 et p. 89-94.

26. Nathalie RichaRd, Inventer la préhistoire. Les débuts de l'archéologie préhistorique en France, Paris, Vuibert et Adapt-Snes., 2008, p. 117-127.

27. Noël Coye, "Une conscience pour le fouilleur : méthodes et techniques de terrain en archéologie préhistorique avant $1950 »$, in Travaux du laboratoire d'anthropologie et de préhistoire des Pays de la Méditerranée occidentale (LAPMO), Aix, université de Provence, 1989, p. 7-28.
28. Noëlle Provenzano, «Fiche terminologie du travail des matières osseuses, du Paléolithique aux âges des métaux », in Denis RAMSEYer (dir.), Matières et techniques. Industrie de l'os préhistorique, vol. 11, Paris, SPF, 2004, p. 29-37.

29. Nous remercions les collaborateurs du Laténium, du SAEF et du Musée national suisse de nous avoir donné accès au matériel d'étude.

30. Les cadres de connaissances et les outils d'analyse en archéologie préhistorique sont en plein développement à la fin du XIX ${ }^{\mathrm{e}}$ siècle. Cette science, pratiquée par des amateurs, n'est pas encore institutionnalisée ni professionnalisée : Marc-Antoine KAESER, «The first establishment of prehistoric science : the shortcomings of autonomy ", in Die Anfünge der ur- und frühgeschichtlichen Archäologie als akademisches Fach (1890-1930) im europäischen Vergleich, Rahden/ Westf, M. Leidorf, vol. 2, 2006, p. 149-160. 